Itinéraires Itinéraires

Littérature, textes, cultures

2020-2 | 2020

Discours animal. Langages, interactions, représentations

\title{
«Le rossignol trouvera bon de parler lui-même de soi-même ». Discours animal et subversion libertine dans les États et Empires de la Lune et du Soleil de Cyrano de Bergerac
}

"Le rossignol trouvera bon de parler lui-même de soi-même". Animal Discourse and Libertine Subversion in Cyrano de Bergerac's États et Empires de la Lune et du Soleil

\section{Aude Volpilhac}

\section{OpenEdition}

Journals

Édition électronique

URL : https://journals.openedition.org/itineraires/8257

DOI : $10.4000 /$ itineraires. 8257

ISSN : 2427-920X

Éditeur

Pléiade

Référence électronique

Aude Volpilhac, « « Le rossignol trouvera bon de parler lui-même de soi-même ». Discours animal et subversion libertine dans les États et Empires de la Lune et du Soleil de Cyrano de Bergerac », Itinéraires [En ligne], 2020-2 | 2020, mis en ligne le 07 janvier 2021, consulté le 25 novembre 2021. URL : http:// journals.openedition.org/itineraires/8257 ; DOI : https://doi.org/10.4000/itineraires.8257

Ce document a été généré automatiquement le 25 novembre 2021.

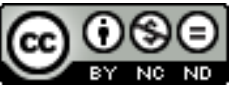

Itinéraires est mis à disposition selon les termes de la licence Creative Commons Attribution - Pas d'Utilisation Commerciale - Pas de Modification 4.0 International. 


\section{«Le rossignol trouvera bon de} parler lui-même de soi-même ". Discours animal et subversion

\section{libertine dans les États et Empires de la Lune et du Soleil de Cyrano de Bergerac}

"Le rossignol trouvera bon de parler lui-même de soi-même". Animal Discourse and Libertine Subversion in Cyrano de Bergerac's États et Empires de la Lune et du Soleil

\section{Aude Volpilhac}

«- Mais alors, selon vous, les oiseaux parleraient?

- Si l'on veut - mais leurs chants sont trop pauvres en modulations distinctes pour qu'on les puisse vraiment qualifier de langage. - Alors les cris des tropis sont-ils assez riches? Nous retombons dans l'histoire du tas de cailloux, soupira Doug. Combien faut-il de mots ou de sons

distincts pour mériter le nom de langage?

- C'est bien là le hic, dit Pop. » (Vercors 1952)

1 Si l'antispécisme est un concept forgé au $\mathrm{xx}^{\mathrm{e}}$ siècle, sa portée polémique et paradoxale émerge déjà $\mathrm{au} \mathrm{XVI}{ }^{\mathrm{e}}$ et au XVII ${ }^{\mathrm{e}}$ siècle, sous la plume notamment de Cyrano de Bergerac. La tradition du voyage imaginaire et satirique dont il s'empare dans les États et Empires de la Lune et du Soleil (1657-1662) propose une exploration à la fois fantaisiste, scientifique et philosophique de l'univers. La fiction y joue un rôle essentiel, notamment dans sa capacité à valider des hypothèses scientifiques jugées encore 
hétérodoxes (l'univers infini, la pluralité des mondes, l'héliocentrisme, etc.) dans un monde où se manifestent de manière tangible les principes d'un matérialisme radical. Mais la fiction, soutenue par une imagination subversive et affranchie de toute contrainte, ouvre aussi le champ des possibles dans l'exercice de la pensée, comme le montre la manière dont Cyrano intègre dans le récit la question du langage animal. De fait, nombreux sont les êtres que nous nommons «non-humains » faute de mieux, que l'auteur hisse au rang de personnages et dont les entretiens organisent l'itinéraire du narrateur. Animaux, végétaux et être conjectural comme le démon de Socrate par exemple ${ }^{1}$ accèdent ainsi à ce statut privilégié d'interlocuteurs et d'actants du récit, la narration leur conférant un rôle et une intériorité en les faisant parler à la première personne. Comme l'a montré Michèle Rosellini (2007), la construction narrative de l'altérité de la parole revêt une fonction polémique complexe au sein des États et Empires de la Lune et du Soleil. Les échanges langagiers constituent en effet des moments charnières du récit car si la rencontre avec l'autre peut être féconde et permettre à l'interlocuteur d'exposer une thèse philosophique hétérodoxe, elle provoque aussi des malentendus, des conflits d'interprétation dont l'issue est parfois dramatique pour le narrateur.

2 La séquence des oiseaux s'inscrit donc dans la perspective plus large de la "pluralisation des langages et des modes de communication" des États et Empires (Parmentier 2004: 220). Les oiseaux font partie de cette galerie d'interlocuteurs nonhumains qui s'expriment à la première personne (du singulier et du pluriel) et que le héros croise principalement lors de son second voyage sur le Soleil. Les effets de cette rencontre sont également ambivalents : si elle apporte un savoir inédit sur l'intériorité animale, elle se clôt par une condamnation à mort du protagoniste humain qui n'en réchappe qu'in extremis. On pourrait d'emblée objecter, au regard de la fiction contemporaine désormais familière depuis le milieu $\mathrm{du} \mathrm{xx}^{\mathrm{e}}$ siècle des procédés stylistiques, narratifs et énonciatifs à même de rendre compte du vécu animal, que Cyrano est loin de faire des oiseaux de véritables sujets de conscience en donnant au lecteur accès à une intériorité singulière qui conserverait toutefois son "altérité incompressible » au regard de l'homme (Morizot 2020 : 67). Cyrano se contenterait ainsi de faire des oiseaux des « locuteurs » (à qui il prêterait artificiellement la parole à des fins argumentatives d'abord anthropocentriques) mais non des " énonciateurs ", c'està-dire des animaux qui seraient la «source de comptes rendus de perception et de pensées représentées", pour reprendre la distinction établie par Sophie MilcentLawson à partir des travaux d'Alain Rabatel sur le point de vue (Milcent-Lawson 2014 et 2018). N'est-il pourtant pas anachronique de reprocher à Cyrano de manquer l'altérité irréductible de l'animal par des procédés analogiques trop anthropomorphiques qui le rabattent sur l'homme, et de lui donner une parole qui ressemble trait pour trait à l'énonciation humaine? N'y voir qu'une forme fruste de ventriloquie reviendrait en effet d'abord à méconnaître la part d'historicité des procédés littéraires de représentation de la vie intérieure animale, dont les techniques n'ont cessé d'évoluer au profit d'une conception de la subjectivité animale envisagée comme foyer sensible de perceptions multiples. L'autre écueil serait de négliger ce que signifie donner la parole à un animal à l'Âge classique. Cyrano accorde en effet délibérément à l'animal, plus qu'une voix, plus encore qu'une parole, un discours rationnel. Car conférer un logos à l'animal - même au moyen de procédés qui semblent rudimentaires aux yeux des modernes - est précisément un acte paradoxal et subversif au XVII siècle, dans la mesure où il remet en cause les opinions communément admises sur la hiérarchie des 
êtres et sur la suprématie de l'homme au sein du vivant. Faire parler l'animal - et, dans le passage qui nous intéresse, des oiseaux - comme l'homme, c'est donc d'abord forcer celui-ci à décentrer son regard en lui montrant que l'animal est un homme comme les autres. À cet égard, le discours animal, dans Les États et Empires de la Lune et du Soleil, cristallise des enjeux d'une part narratifs, stylistiques et énonciatifs et, de l'autre, polémiques, philosophiques et éthiques. Le discours des oiseaux est en effet à la fois un élément à part entière du récit fictionnel et un philosophème qui n'a d'autre but que d'ébranler une certaine conception de l'homme et de l'animal.

\section{La séquence des oiseaux dans les États et Empires du Soleil}

La séquence que nous avons détachée repose sur trois des épisodes qui tissent la trame narrative du voyage du narrateur-personnage Dyrcona sur le Soleil: le récit des aventures du rossignol amoureux, le discours polémique et philosophique du phénix sur le langage des oiseaux et enfin le procès du héros au royaume des oiseaux, ces derniers dressant alors un réquisitoire contre l'espèce humaine, accusée de toutes les violences, réelles et symboliques, à leur égard. La cohérence de cette séquence est produite d'abord par l'unité du personnel dramatique formé par les oiseaux, répartis en de nombreuses espèces: le rossignol, le phénix, l'aigle, les pies, les geais, les étourneaux, l'hirondelle, la colombe, la perdrix, l'orfraie, l'autruche, les condors et les griffons, les corbeaux, les chouettes, les oiseaux de paradis, les hérons, les cormorans, les tourterelles et un perroquet. Autant d'oiseaux réels et mythologiques, familiers et exotiques, paradigmatiques et symboliques ${ }^{2}$, dont la variété de statuts annonce d'emblée l'enjeu profond du récit tout autant que le plaisir d'offrir un tableau foisonnant des espèces naturelles. Dans cet épisode, les oiseaux, seuls ou en groupe, remplissent des rôles de plus ou moins grande importance dans l'action, qu'ils aident le héros, comme le perroquet César qui le sauve in extremis de la mort (Cyrano : 274), ou lui nuisent, comme les hérons et les cormorans dont les longs cous servent à le ligoter lorsqu'il est fait prisonnier (Cyrano: 272). Le récit s'attarde sur les aventures de certains oiseaux, qui sont alors plus individualisés, comme le rossignol ou le perroquet. Ils sont alors parfois dotés d'un nom qui peut être hérité de la tradition comme la pie Margot, ou d'un sobriquet, comme la perdrix surnommée Guillemette la Charnue. L'illusion de personne, éventuellement amorcée par le nom propre, est ensuite parachevée par l'exercice de la parole.

4 Le langage se manifeste d'emblée dans sa composante linguistique: si le phénix polyglotte connaît le français, la plupart des oiseaux choisis pour le procès de Dyrcona sont francophones parce qu'ils ont auparavant vécu en France, le narrateur prenant soin de le préciser dans un souci de vraisemblance incontestablement comique (Nédelec 2004). Ce langage est non seulement oral, mais écrit, le procès organisé par les oiseaux exigeant d'abord la rédaction de l'acte d'accusation "sur l'écorce d'un cyprès " (Cyrano : 256) puis la lecture de la sentence, prononcée symboliquement par l'orfraie (Cyrano: 269). Par ailleurs, le langage des oiseaux se déploie selon une très large gamme de types de discours qui s'étend du discours raisonné au discours irrationnel, de l'exposé philosophique à la vindicte populaire, en passant par la rhétorique judiciaire sous différentes formes, qu'il s'agisse du réquisitoire, de la défense, du jugement. La narration détaille même parfois les conditions d'énonciation et de réception du 
discours, en présentant une situation de dialogue plus intime et plus personnel où le corps singulier de l'animal est évoqué : tandis que la pie, maîtrisant l'art oratoire de la consolatio, met «le bec à l'oreille du héros", le perroquet vient «de ces deux ailes accoler le visage » du narrateur pour lui parler (Cyrano: 273). Que ces précisions fassent sourire n'empêche pas qu'elles mettent au centre de la fiction une parole animale incarnée, dans ses similitudes et ses différences avec celle de l'homme. Elles ménagent surtout, dans les interstices du récit, la question philosophique du discours animal et du rapport complexe entre ce discours et le corps qui le profère.

\section{Une parole confisquée : l'âme du rossignol}

5 L'aventure du rossignol amoureux - la première du cycle des oiseaux - s'apparente d'abord à une mise en récit de la thèse de l'âme des bêtes. Mais cet épisode repose sur un ressort narratif inattendu. Le héros-narrateur vient de faire la rencontre d'un être solaire extraordinaire, roi d'un peuple capable comme lui de toutes les métamorphoses physiques. Dyrcona, le roi et son peuple se trouvent alors émus aux larmes par le chant mélancolique et amoureux d'un rossignol. L'être solaire, les poumons trop comprimés par sa petite taille pour s'exprimer aisément, s'apprête à céder la parole à l'oiseau afin que celui-ci raconte en personne son aventure : « le rossignol trouvera bon de parler luimême de soi-même" (Cyrano: 238). Malgré cette invite à prendre en charge personnellement le récit de sa propre vie, l'oiseau ne s'exprime pas ; plus exactement, la prise de parole n'a pas lieu :

Je [c'est Dyrcona qui parle] lui répliquai que je n'avais point encore assez l'habitude au langage d'oiseau; que véritablement un certain philosophe, que j'avais rencontré en montant au soleil, m'avait bien donné quelques principes généraux pour entendre celui des brutes; mais qu'ils ne suffisaient pas pour entendre généralement tous les mots ni pour être touché de toutes les délicatesses qui se rencontrent dans une aventure telle que celle-là. (Cyrano : 239)

La prise de parole à la première personne du rossignol, dont le récit suggère qu'elle est possible, demeure toutefois à l'état de virtualité. Pour autant, ce vide textuel est paradoxalement éloquent parce qu'il provient du refus du narrateur humain lui-même qui incrimine son incapacité à comprendre le langage d'oiseau, par manque de pratique et de familiarité, mais surtout par manque de finesse et de subtilité, voire de sensibilité.

6 Le coup de théâtre orchestré par la fiction - un oiseau peut parler - est d'emblée renversé par l'effet déceptif de la suite du récit qui déjoue les attentes du lecteur. S'il s'agit d'une part de souligner que l'inintelligibilité du langage animal est le fait de l'infirmité humaine ${ }^{3}$, de l'autre, la frustration du lecteur ménage, pourrait-on dire, la punition de l'homme pour son orgueil : sa présomption le prive d'un chant qui aurait été sans doute extraordinaire. La narration propose toutefois de compenser ce silence par le truchement de l'être solaire. Ce dernier insiste toutefois sur la perte irrémédiable qu'engendre un récit qui ne peut qu'imparfaitement et partiellement "traduire" l'expérience subjective du rossignol à l'intention de Dyrcona : « Hé bien, dit-il, puisque tu le veux, tes oreilles ne seront pas simplement sevrées des belles chansons du rossignol, mais de quasi toute son aventure, de laquelle je ne te puis raconter que ce qui est venu à ma connaissance. Toutefois tu te contenteras de cet échantillon [...]» (Cyrano : 239).

7 L'être solaire raconte donc au narrateur comment, alors qu'il avait pris l'apparence d'un rossignol, un véritable rossignol s'éprit progressivement de lui. Le quiproquo 
s'aggrave quand l'être solaire, entouré de ses sujets ayant pris eux-mêmes l'apparence d'aigles, ne veut pas détromper le véritable rossignol pour jouir de la beauté de son chant - manigance fallacieuse qui est pourtant la condition de possibilité de la démonstration de la sensibilité animale. Il entretient alors le rossignol dans l'erreur, lui laissant croire qu'il est prisonnier des aigles et qu'il est sans doute condamné à mourir. Cette tromperie dure assez longtemps pour permettre au récit de déployer toute la gamme des sentiments qu'éprouve le véritable oiseau. Le passage détaille ainsi les différentes phases de la cristallisation amoureuse, qui naît de l'empathie et de la générosité - il croit le faux rossignol prisonnier (Cyrano : 242) - et que renforce la situation désespérée de l'être aimé pour lequel le rossignol est prêt à sacrifier héroïquement sa vie. Et même quand l'être solaire lui avoue la fourberie, le rossignol, abusé par sa passion, se refuse à le croire. Cette aventure prouve ainsi l'existence d'une vie intérieure - même s'il s'agit du rossignol, symbole lyrique - constituée d'états psychologiques subtils et dignes d'idéaux courtois et galants ${ }^{4}$. En expliquant le comportement de l'oiseau comme le résultat de son caractère et de ses valeurs morales, Cyrano suggère que l'agir animal ne peut se réduire à une mécanique. Ce faisant, il attribue à son personnage les qualités d'une personne humaine. De fait, les sentiments du rossignol prennent la forme d'une véritable passion amoureuse : «Nous gazouillions l'un après l'autre réciproquement l'histoire en musique de nos mutuelles amours " (Cyrano: 242). La langue devient elle aussi poétique et la régularité rythmique vient renforcer ce moment de grâce - même s'il est sans doute aussi humoristique - qui culmine dans l'évocation du duo amoureux, manifestation tangible de l'âme des bêtes. La profondeur et la complexité psychologiques du rossignol en font un être singulier, un individu dont la passion mérite d'être racontée au même titre que celle de nombreux autres personnages humains de la littérature. L'artifice du discours rapporté (Cyrano : 242) permet ainsi d'entrevoir la possibilité d'une subjectivité animale.

8 Attribuer la parole à un «non-humain » n'est pas seulement un procédé narratif propre au voyage fictionnel ; la question du langage animal est au cœur des entretiens de cette séquence. Énonciation et énoncé convergent donc vers la même démonstration, le discours animal constituant le propos central du phénix dans le deuxième épisode de cette séquence dans un effet de mise en abyme où l'oiseau fabuleux prend la parole pour apprendre au narrateur que les oiseaux sont capables de langage. Le propos redouble de manière discursive ce qui a lieu dans le récit: le changement de statut de l'animal, érigé au rang de personnage. À la question que soulève un tel récit - à partir de quand peut-on parler de personnage ?-, on peut déjà suggérer que certains oiseaux ne sont pas tant dotés de parole qu'ils en sont doués : telle est du moins la thèse qui confère sa cohérence polémique à cet épisode et qui permet de faire le départ entre la fiction cyranienne et la fable allégorique où des animaux parlent pour faire le procès des hommes. En d'autres termes, l'artifice littéraire rend possible ce que l'observation empirique ne peut et ne pourra sans doute jamais apporter : l'accès à l'intériorité des bêtes. Or, qu'elles possèdent un véritable langage, une raison et une conscience, c'est ce qui leur est communément dénié au XVII ${ }^{\mathrm{e}}$ siècle.

Ce changement de statut littéraire repose en effet sur une conception hétérodoxe de l'animal et, plus largement, du vivant, qui révèle l'intention polémique de Cyrano de troubler la représentation ontologique des existants alors dominante. Ce philosophème est identifié d'emblée par un lecteur contemporain éclairé, familier des controverses philosophiques de son temps. Cyrano met ainsi en fiction une polémique philosophique 
ancienne et renouvelée par Descartes au XvII ${ }^{\mathrm{e}}$ siècle ${ }^{5}$. Schématiquement, la controverse sur le langage animal se cristallise sur trois points : le lien entre langage et raison, le statut ontologique de l'homme et de l'animal et ses conséquences éthiques.

\section{« Un discours au dedans ${ }^{6}$ »}

10 Dès l'Antiquité, la controverse sur le langage des animaux, loin de se réduire à la question de l'expression et de la communication, est plus largement liée à celle de la pensée et de la raison animales. Si cette querelle philosophique sur l'intelligence des bêtes a une histoire dont il est impossible ici de rendre compte exhaustivement (Gontier 1999 et 1998)7 ${ }^{7}$, tout au plus peut-on rappeler qu'elle s'est fixée à l'époque hellénistique dans la polémique qui opposa deux écoles, celle des stoïciens et celle des académiciens. Comme le montre Thierry Gontier, les premiers refusent la raison à l'animal, attribut exclusif de l'homme et, pour démontrer cette différence, distinguent deux types de signes de la raison: le langage et les actions, le premier, que seul possèdent les hommes, témoignant de la raison proférée (logos prophorikos), c'est-à-dire de la capacité à communiquer ses pensées. La conséquence de cette distinction entre l'homme et l'animal est un anthropocentrisme radical: l'homme se trouve donc au sommet d'une hiérarchie entre les êtres qui justifie qu'il jouisse et abuse de tout ce qui l'entoure. Contre cette conception anthropocentrique et dogmatique - pour leurs adversaires, on ne peut établir un savoir véritable sur l'intériorité des animaux -, les Académiciens de la nouvelle Académie sceptique nient, d'une part, qu'il soit possible de rendre compte de leur vécu, qui demeure inaccessible aux hommes, et, de l'autre, défendent l'idée que les animaux manifestent leur raison, par leurs actions, mais aussi par leur langage (Gontier $1998: 32$ ).

Dans sa forme moderne, la polémique est d'abord renouvelée par Montaigne. Le célèbre chapitre 12 du livre II des Essais, «L'apologie de Raimond Sebond », joua un rôle majeur dans le bouleversement des frontières idéologiques entre l'homme et l'animal en donnant un nouvel élan à la tradition sceptique antique. Puis la querelle sur l'âme des bêtes, déclenchée par Descartes, et dont Cyrano se fait ici l'écho, s'est amplifiée dans la seconde moitié du siècle. La théorie de l'animal-machine, théorie « cartésienne » si l'on comprend par ce terme le fait qu'elle est entérinée par les héritiers de Descartes plus que par Descartes lui-même, plus nuancé sur ce point (Larrère 2014), repose sur le principe selon lequel le langage constitue le signe repérable qui permet d'affirmer la présence (ou l'absence) de toute forme de conscience et de raison (Descartes 1637). À cet égard, le "discours animal» est un oxymore pour les cartésiens, car, selon eux, l'observation des animaux prouve qu'on ne peut confondre langage et expression, critère qui distingue donc radicalement l'homme du reste des vivants.

Ce n'est donc pas un hasard si Cyrano confie à une pie et un perroquet de véritables rôles dans le récit. Ces espèces étaient déjà "canoniques" dans la querelle sur le langage animal dès l'Antiquité, en raison de leur capacité d'imitation; elles furent à leur tour convoquées par Montaigne, pour lequel la manière dont elles apprennent à articuler des syllabes est la preuve d'un " discours au dedans ${ }^{8}$ " (Montaigne : 195), c'està-dire d'une capacité intellectuelle. Descartes va mobiliser à son tour ces oiseaux mais pour mieux contrer l'argument sceptique en distinguant l'imitation phonique du langage articulé (Descartes 1637 : 57). En mettant en récit cette polémique, Cyrano, nettement anti-cartésien sur ce point (Torero-Ibad 2009), rend possible au contraire, 
par le biais de la fiction, l'idée que les animaux ont part au logos. C'est sans doute parce que Cyrano est d'abord anti-chrétien et matérialiste : le monde sans Dieu des libertins repose sur le principe que tous les êtres vivants ont en partage leur commune appartenance à la matière, à l'opposé du dogme religieux selon lequel l'homme, seul être vivant doté d'une âme, domine légitimement une nature qui aurait été créée pour lui.

13 Faire parler les oiseaux signifie donc qu'ils sont capables de penser et de raisonner, comme le souligne clairement la présence du lexique du discours qui organise la séquence. Ils possèdent donc un logos au double sens de langage et de raison, le langage manifestant les opérations de la raison vocalisées par les organes physiques. C'est du moins la thèse que défend le phénix. Certes, on pourrait objecter que, comme il s'agit d'un animal fabuleux, il n'est pas surprenant qu'il puisse parler - telle serait la loi de l'univers fantastique-; toutefois, cette identité mythologique est volontairement différée dans le récit et n'est révélée qu'après le « discours » (Cyrano : 250) de l'oiseau. Par conséquent, le lecteur pense spontanément qu'il s'agit d'un oiseau qui parle. Dyrcona, que les conditions particulières de la vie solaire rendent plus subtil, découvre alors que l'oiseau «parle en chantant»: «je remarquai distinctement les syllabes, les mots et le discours qu'il articula » (Cyrano : 250). Le narrateur met d'emblée l'accent sur l'un des aspects de la polémique sur le langage animal: la notion de langage articulé. Le $\mathrm{XvII}^{\mathrm{e}}$ siècle hérite en effet d'une longue tradition selon laquelle si les hommes et les animaux ont en commun la voix (comprise comme émission sonore), les premiers se distinguent en revanche des seconds par l'exercice de la parole, définie par l'articulation, le mot articulé (par la maîtrise du discontinu où la discontinuité devient signifiante par la succession des syllabes) servant à expliquer la pensée (Le Guern 1991). C'est donc le critère de l'articulation qui, communément, permet de faire le départ entre le langage des hommes et les sons animaux et c'est cette frontière symbolique que le récit cherche à fissurer.

Tel est aussi l'enjeu de l'exposé philosophique pris en charge par le phénix, et nourri des arguments classiques en faveur de la thèse du langage animal : ne pas comprendre les animaux n'implique pas pour autant qu'ils ne sont pas raisonnables (Cyrano : 251). Le langage est diversement maitrisé par les oiseaux, comme c'est aussi le cas pour les hommes : « tout ainsi qu'il se rencontre des oiseaux qui ne disent mot, quelques-uns qui gazouillent, d'autres qui parlent, il s'en rencontre encore de plus parfaits qui savent user de toutes sortes d'idiomes..." (Cyrano: 252). Loin de tracer une frontière hermétique entre les hommes et les animaux, le langage est d'abord une question de compétence innée et acquise, inégalement répartie dans une même espèce.

\section{Discours animal et procès de l'homme}

Affirmer que les animaux ont un langage signifie donc que le logos et les sentiments ne sont pas l'apanage des hommes. Cyrano peut alors, dans la lignée du scepticisme antique revivifié par Montaigne, faire la critique de la vanité humaine. C'est ainsi que l'on peut interpréter les scènes de rencontre et d'écoute entre le personnage-narrateur et les oiseaux: celui-ci entend dans un premier temps des bruits confus dont il comprend seulement par la suite qu'il s'agit d'un véritable langage ${ }^{9}$. Déjà lors du voyage sur la Lune, le narrateur avait rencontré des étrangers - il se croit sur la Lune mais va comprendre par la suite qu'il a atterri en Nouvelle-France - dont la langue lui avait 
paru au premier abord "non articulée", au point de la confondre avec «le gazouillement enroué d'un muet » (Cyrano : 12). Si le motif du langage articulé permet de dénoncer ici l'ethnocentrisme (Parmentier 2004: 222), il devient sur le Soleil le moyen de condamner l'anthropocentrisme. La question de l'inintelligibilité du langage animal, résolue de la sorte, met en valeur les faiblesses humaines, notamment l'incapacité spontanée de l'homme à entendre le langage animal ; elle permet en outre une remise en cause du phonologocentrisme, c'est-à-dire le primat du langage articulé sur les autres formes de communication.

Dans sa forme antique, la controverse sur le langage animal était déjà subordonnée à la définition de l'homme (Gontier $1998: 14$ ). Après Descartes, c'est l'héritage aristotélicien et le christianisme qui forment la seconde cible de Cyrano. En accordant la parole aux animaux, il rejette vigoureusement l'échelle des êtres orientée vers le logos d'Aristote (Gontier 1998 : 31) et nie la prétendue spécificité ontologique de l'homme en affirmant qu'il n'y a pas de différence de nature, mais seulement une différence de degré entre tous les êtres vivants. Tel est le décentrement radical opéré par la séquence des oiseaux dans les États et Empires de la Lune et du Soleil et dont l'enjeu est profondément éthique : il s'agit bien, in fine, de réapprendre à voir autrement les êtres naturels en se déprenant de nos catégories mentales. La critique de l'anthropocentrisme, portée par le phénix mais surtout par le réquisitoire des oiseaux contre l'homme, a pour corollaire la condamnation de la domination que celui-ci prétend exercer en toute légitimité sur les «non-humains ». En effet, dans le troisième épisode, le héros humain se retrouve prisonnier des oiseaux qui l'accusent de nuire à leur espèce. Il est donc jugé avant d'être condamné. Le renversement de point de vue, que favorise la prise de la parole des oiseaux à la première personne, rend l'accusation de «barbarie » (Cyrano : 265) plus efficace encore :

Voilà le bel effet de cette fantastique monarchie et de cet empire si naturel de l'homme sur les animaux et sur nous-mêmes, car son insolence a été jusque-là. Cependant, en conséquence de cette principauté ridicule, il s'attribue tout joliment sur nous le droit de vie et de mort ; il nous dresse des embuscades, il nous enchaîne, il nous jette en prison, il nous égorge, il nous mange, et, de la puissance de tuer ceux qui sont demeurés libres, il fait un prix à la noblesse. Il pense que le soleil s'est allumé pour l'éclairer à nous faire la guerre; que Nature nous a permis d'étendre nos promenades dans le ciel, afin seulement que de notre vol il puisse tirer de malheureux ou favorables auspices; et quand Dieu mit des entrailles dedans notre corps, qu'il n'eut intention que de faire un grand livre où l'homme pût apprendre la science des choses futures. (Cyrano : 266)

L'argument relativiste ne conduit toutefois pas Cyrano à une représentation idéalisée des animaux.

\section{Ambivalence du discours animal}

17 Les types de discours sont variés dans la séquence des oiseaux, et se distribuent sur un large éventail qui va de l'exercice de la raison à l'absence totale de rationalité. Les oiseaux en effet, capables d'argumentation logique ou de raisonnement philosophique, peuvent aussi faire un usage dévoyé de la raison, comme les hommes. C'est du moins toute l'ambivalence de l'épisode du procès du héros-narrateur. Le long réquisitoire contre la supériorité prétendue des hommes s'achève ainsi sur ces mots :

Hé bien! ne voilà pas un orgueil tout à fait insupportable ? Celui qui l'a conçu pouvait-il mériter un moindre châtiment que de naître homme? Ce n'est pas 
toutefois sur quoi je vous presse de condamner celui-ci. La pauvre bête n'ayant pas comme nous l'usage de raison, j'excuse ses erreurs quant à celles que produit son défaut d'entendement. (Cyrano : 266-267)

C'est ironiquement au moment où les oiseaux affirment la supériorité de leur raison sur celle des hommes qu'ils en manquent le plus et que celle-ci se met à divaguer. Les accusations portées contre l'espèce humaine, aussi légitimes soient-elles, sont contrebalancées par la conviction, forte chez la majorité des oiseaux, de la supériorité ontologique de leur espèce sur les hommes comme du fait que la nature a été faite pour $e^{10}{ }^{10}$. En outre, l'ornithocentrisme du réquisitoire, qui montre les difficultés que tout esprit rencontre pour bien penser, est renforcé dans le récit par l'insistance sur les réactions violentes de la "populace » (Cyrano : 267). La vindicte populaire est en effet évoquée par un large spectre de termes qui figurent un usage dévoyé du langage. Ce dernier s'apparente davantage au bruit et à la fureur: autant de "cris », de « murmures» (Cyrano : 268), parmi lesquels un oiseau « claque du bec » (Cyrano : 267) pour manifester son âpre désir de vengeance. Si le langage gagne en expressivité ce qu'il perd en rationalité, il s'agit surtout de dénoncer les méfaits d'une parole anonyme et collective, soumise aux passions primaires échauffées par l'effet d'entraînement propre à la foule.

18 En réalité, l'horizon d'attente utopique suggéré par la progression narrative de la séquence des oiseaux, dans la mesure où cet ultime épisode dans leur royaume succède à celui du rossignol amoureux - qui prouve la grandeur d'âme et la profondeur psychologique de l'animal - et celui du phénix - qui pose l'existence du langage animal-, est déçu. Au contraire, l'emprisonnement du héros et sa condamnation à mort, thèmes par ailleurs structurants des deux voyages sur la Lune et sur le Soleil, suggèrent qu'il serait réducteur de rabattre de manière univoque cette séquence narrative sur la thèse philosophique du langage des bêtes sans prendre la mesure de la complexité et de l'ambivalence de la fiction littéraire. Admettre que les animaux ont une forme d'intelligence et de langage, c'est accepter en contrepartie que, comme les hommes, certains d'entre eux font un mauvais profit de la raison et que tous ne sont pas capables de l'exercer à bon escient. Telle est également la position élitiste propre aux libertins du XVII ${ }^{\mathrm{e}}$ siècle (Moreau 2007) : aussi trouve-t-on chez les oiseaux, comme chez les hommes, des "sages » à la parole mesurée qui s'opposent à " la commune ", soumise à l'irrationalité et à l'intolérance. En somme, remettre en cause la hiérarchie ontologique entre les êtres vivants n'est pas incompatible avec la conviction élitiste d'une hiérarchie des tempéraments au sein d'une même espèce.

\section{Une éthique des rapports entre les vivants}

19 Que ce récit soit à la première personne ne relève pas seulement d'un procédé classique du voyage imaginaire qui met le héros-narrateur en son centre. Si Dyrcona constitue le fil qui organise la trame des aventures et favorise la mise en scène de l'émerveillement, il ne se contente pas d'observer passivement les événements qu'il raconte. Ainsi, lors de son séjour au royaume des oiseaux, le héros-narrateur ne fait pas seulement une découverte intellectuelle sur les capacités cognitives et sur l'âme des bêtes. Sa rencontre avec les oiseaux - aussi négative soit-elle lors de son incarcération -, loin d'être abstraite et désincarnée, relève de l'expérience sensible. En effet, le corps du personnage joue un rôle central dans le récit (Rosellini 2005 : 105-113). À l'arrivée du 
phénix par exemple, le narrateur détaille les réactions physiologiques et émotionnelles que provoque en lui sa présence :

Il fut longtemps à voler dans la nue, et je me tenais tellement collé à tout ce qu'il devenait, que mon âme s'étant toute repliée et comme raccourcie à la seule opération de voir, elle n'atteignit presque pas jusqu'à celle d'ouïr, pour me faire entendre que l'oiseau parlait en chantant.

Ainsi peu à peu débandé de mon extase, je remarquai distinctement les syllabes, les

mots et le discours qu'il articula. (Cyrano : 250)

Cette rencontre, de l'ordre de la fusion charnelle, est justifiée par les lois physiques propres à l'univers solaire. En décuplant les pouvoirs de l'imagination, elles rendent possible un rapport immédiat et érotique entre tous les êtres d'une même nature matérielle qui peut éventuellement faire l'économie du langage articulé ${ }^{11}$. Cyrano associe souvent dans les États et Empires du Soleil la question de la communication à la présence du corps et de ses métamorphoses (Parmentier $2004: 220$ ). Le matérialisme radical de Cyrano, loin de se définir de manière abstraite, se prolonge ici dans un sensualisme qui engage aussi un rapport éthique au monde des existants auquel la fiction donne une forme tangible et concrète. Cette scène pourrait renvoyer à une forme d'expérience mystique car il s'agit bien d'une sorte de révélation transcendante qu'apporte le phénix annonçant la bonne nouvelle du langage et de l'intériorité animales ; pour autant, le motif demeure parodique dans le monde solaire sans Dieu. Si l'on peut y voir aussi, dans une perspective métalittéraire, le récit d'une expérience esthétique, l'arrivée du phénix figurant métaphoriquement la puissance du récit poétique, défini par l'enargeia - catégorie rhétorique selon laquelle le langage littéraire rend visible, révèle l'évidence d'une représentation -, l'enjeu d'un tel passage est aussi assurément éthique. L'érotisme manifeste de cette communion immanente et puissante de l'homme avec l'oiseau fait signe vers l'idée d'une communauté des vivants puisque l'homme, comme tous les êtres naturels, relève de la seule matière. Or, c'est précisément cette appartenance commune au règne de la matière qui justifie les affinités électives entre le héros-narrateur et l'oiseau.

La fiction de la «langue matrice ", racontée dans un épisode qui précède l'arrivée de Dyrcona sur le Soleil, renforce cette idée d'une communauté des êtres naturels. En effet, alors que le narrateur comprend comme par miracle "plus vite et plus intelligiblement [la langue] du petit homme » qu'il rencontre sur la macule solaire, celui-ci lui en révèle les raisons :

Il m'expliqua quand je me fus enquis d'une chose si merveilleuse, que dans les sciences il y avait un vrai, hors lequel on était toujours éloigné du facile ; que plus un idiome s'éloignait de ce vrai, plus il se rencontrait au-dessous de la conception et de moins facile intelligence.

« De même, continuait-il, dans la musique ce vrai ne se rencontre jamais, que l'âme aussitôt soulevée ne s'y porte aveuglément. Nous ne le voyons pas, mais nous sentons que Nature le voit; et sans pouvoir comprendre en quelle sorte nous en sommes absorbés, il ne laisse pas de nous ravir, et si, nous ne saurions remarquer où il est. Il en va des langues tout de même. Qui rencontre cette vérité de lettres, de mots, et de suite, ne peut jamais en s'exprimant tomber au-dessous de sa conception: il parle toujours égal à sa pensée; et c'est pour n'avoir pas la connaissance de ce parfait idiome que vous demeurez court, ne connaissant pas l'ordre ni les paroles qui puissent expliquer ce que vous imaginez». (Cyrano: 217-218).

Cette langue mythique, capable de toucher à l'essence des choses et de rendre possibles l'expression de la vérité et sa communication télépathique immédiate est, certes, aussi 
rapidement évoquée qu'elle sera oubliée dans la suite du récit, ne jouant de fait pas de rôle dans la séquence au royaume des oiseaux. Pour autant, elle suggère elle aussi l'existence d'une langue unique de la nature à même d'établir une véritable communauté égalitaire des vivants qui fait fi des différences entre les règnes et nie les hiérarchies. En effet, comme le remarque Michèle Rosellini, la langue-matrice est une "langue anti-biblique » parce qu'elle « refuse d'être un instrument de domination du monde » (Rosellini 2007 : 285).

Il s'agit donc bien, d'une certaine manière, selon les termes de Philippe Descola, de faire voler en éclats « l'ancienne opposition au sein du vivant entre les êtres de langage et les autres » (Descola 2017 : 17). Pour Cyrano, remettre en cause cette frontière ontologique imaginaire passe par l'idée que tous les vivants possèdent un langage. En effet, de manière radicale, le libertin porte ce paradoxe à son comble en donnant logiquement naturellement pourrait-on dire -, la parole au végétal au même titre qu'il l'a reconnue à l'animal. En faisant parler un chou désespéré d'être décapité puis un chêne voluptueux, les États et Empires de la Lune et du Soleil cherchent à ruiner toute présomption humaine et renversent les paradigmes aristotélicien, chrétien et cartésien. Après son passage au royaume des oiseaux, Dyrcona découvre ainsi, dans un épisode symétrique à celui que nous avons étudié, que les arbres ont également un langage dont l'inintelligibilité première révèle davantage encore l'infirmité humaine :

Ainsi étendu à l'ombre de ces arbres, je me sentais inviter au sommeil par la douce fraîcheur et le silence de la solitude, quand un bruit incertain de voix confuses, qu'il me semblait entendre voltiger autour de moi, me [réveilla] en sursaut.

Le terrain paraissait fort uni et n'était hérissé d'aucun buisson qui pût rompre la vue; c'est pourquoi la mienne s'allongeait fort avant entre les arbres de la forêt. Cependant le murmure, qui venait à mon oreille, ne pouvait partir que de fort proche de moi ; de sorte que, m'y étant rendu encore plus attentif, j'entendis fort distinctement une suite de paroles grecques; et, parmi beaucoup de personnes qui s'entretenaient, j'en démêlai une qui s'exprimait ainsi [...]. (Cyrano : 274-275)

En affirmant que la plante ressent et pense, en d'autres termes qu'elle est pourvue d'une âme capable du cogito, Cyrano envisage la relation entre les existants non plus en termes duels, mais en y réintroduisant le tiers traditionnellement exclu qu'est la plante (Brancher 2015).

\section{Conclusion}

Cyrano de Bergerac, dans la séquence des oiseaux des États et Empires du Soleil, met donc en fiction une position philosophique et polémique qui n'est pas nouvelle: le récit élabore ainsi les conditions de possibilité d'un langage animal, signe incontestable pour l'époque de la raison. Cyrano leur reconnaît aussi une âme, mue par la loi du désir propre au vivant. Toutefois, point d'utopie sur le soleil : la raison animale, comme celle des hommes, peut dérailler, de même que les passions peuvent aliéner l'individu. Certes, il s'agit toujours, in fine, de faire choir l'homme du piédestal où il s'est indûment installé depuis des siècles; mais cette subjectivisation animale, rendue possible par la fiction, implique une éthique qui propose de refonder les relations qui doivent se nouer entre les vivants. Ébranler la hiérarchie des êtres au moyen d'un matérialisme radical implique donc aussi d'envisager à nouveaux frais le rapport de l'homme à l'animal et plus largement à la nature. C'est en tout cas ce que favorise l'espace littéraire, capable, en raison de sa malléabilité, de reconfigurer librement ces rapports. Pour autant, point non plus ici d'utopie, mais un souci fondamental du décentrement du regard, au 
moment où le monde végétal et les animaux sont en passe de devenir, sous le règne de Louis XIV, un outil politique au service de l'absolutisme notamment par la mise en spectacle d'une nature domestiquée (Sahlins 2017).

Une séquence du roman, de moindre intensité narrative, rapporte ainsi la manière dont Dyrcona fait pendant quelque temps route commune vers le royaume des oiseaux avec le rossignol :

Je voyageai sur cette espérance, pour le moins trois semaines, avec toutes sortes de contentement, si je n'eusse eu que mes oreilles à satisfaire; car le rossignol ne me laissait point manquer de musique ; quand il était plus las, il venait se reposer sur mon épaule; et quand je m'arrêtais, il m'attendait. À la fin j'arrivai dans une contrée du royaume de ce petit chantre, qui alors ne se soucia plus de m'accompagner. (Cyrano : 249)

Tel est peut-être le seul idéal possible du rapport à l'animal proposé en creux par le texte: un compagnonnage et une attention réciproque, véritable "intimité sans proximité " (Despret 2018: 12). L'être solaire qui, en raison de ses pouvoirs extraordinaires, s'exprime à la place du rossignol dont il comprend l'âme, pourrait ainsi représenter le rôle de l'écrivain qui découle de cette conception de la communauté des êtres vivants : tout en se faisant le truchement d'un animal, il sait qu'il ne peut qu'en suggérer partiellement le vécu. Pour autant, cette incomplétude ne manque pas son objet, mais stimule sa quête. À la manière du traducteur de poésie, l'écrivain se doit de «traduire l'intraduisible » (Morizot $2020: 67$ ).

\section{BIBLIOGRAPHIE}

Brancher, Dominique, 2015, Quand l'esprit vient aux plantes. Botanique sensible et subversion libertine (XVI ${ }^{e}$-XVII siècle), Genève, Droz.

Descola, Philippe, 2017, préface à E. Kohn, Comment pensent les forêts, Bruxelles, Zones sensibles.

Despret, Vinciane, 2018, préface à B. Morizot, Sur la piste animale, Arles, Actes Sud.

Fontenay, Élisabeth de, 1998, Le Silence des bêtes. La philosophie à l'épreuve de l'animalité, Paris, Fayard.

Gengoux, Nicole, 2015, Une lecture philosophique de Cyrano : Gassendi, Descartes, Campanella, trois moments du matérialisme, Paris, Champion.

Gontier, Thierry, 1998, De l'homme à l'animal. Paradoxes sur la nature des animaux. Montaigne et Descartes, Paris, Vrin.

Gontier, Thierry, 1999, L'Homme et l'animal. La philosophie antique, Paris, PUF.

Laugaa, Maurice, 1973, «Cyrano: Sound and Language », Yale french Studies, n 49, p. 199-201.

Larrère, Catherine, 2014, "Que savons-nous des animaux ? Machines ou êtres sensibles ? ", dans V. Despret et R. Larrère (dir.), Les Animaux : deux ou trois choses que nous savons d'eux, Paris, Hermann, p. 61-82. 
Le Guern, Odile, 1991, «Cureau de La Chambre et les sciences du langage à l'âge classique », Corpus, $\mathrm{n}^{\circ}$ 16-17, « L'âme des bêtes », p. 17-25.

Milcent-Lawson, Sophie, 2014, « Points de vue et discours animaux dans le romanesque gionien ", dans A. Romestaing (dir.), Mondes ruraux, mondes animaux. Le lien des hommes avec les bêtes dans les romans rustiques et animaliers de langue française (XXe-XXI ${ }^{e}$ siècles), Dijon, EUD, coll. « Écritures », p. 61-72.

Milcent-Lawson, Sophie, 2018, « Du chien confident à l'animal sujet de conscience. Alice Ferney, Dans la guerre ", Actes du colloque « La parole aux animaux. Conditions d'extension de l'énonciation », [En ligne], https://www.fabula.org/colloques/document5380.php.

Moreau, Isabelle, 2007, « Guérir du sot ». Les stratégies d'écriture des libertins à l'âge classique, Paris, Champion.

Morizot, Baptiste, 2020, Manières d'être vivant, Arles, Actes Sud.

Nédelec, Claudine, 2004, « Un monstre qui n'est que de langues », Lectures de Cyrano de Bergerac. Les États et Empires de la Lune et du Soleil, Rennes, PUR, p. 147-162.

Parmentier, Bérengère, 2004, « Parler sans la langue. Langages et corps dans Les États et Empires de Cyrano de Bergerac », Littératures classiques, Cyrano de Bergerac, Les États et Empires de la Lune et du Soleil, $\mathrm{n}^{\circ}$ 53, supplément, p. 219-236.

Rosellini, Michèle, 2005, Cyrano de Bergerac, Les États et Empires de la Lune et du Soleil, Neuilly, Atlande.

Rosellini, Michèle, 2007, «Paroles de l'Autre monde : les usages hétérodoxes du dialogue dans les États et Empires de Cyrano de Bergerac ", Cahiers du GADGES $n^{\circ} 5$ : Parole de l'Autre et genres littéraires (XVI ${ }^{e}-\mathrm{XVII}^{e}$ siècle). Illustrations, interactions, subversions, Pierre Servet et Marie-Hélène Servet-Prat (dir.), Lyon, p. 273-294.

Sahlins, Peter, 2017, 1668: The Year of the Animal in France, New York, Zone Books.

Torero-Ibad, Alexandra, 2009, Libertinage, science et philosophie dans le matérialisme de Cyrano de Bergerac, Paris, Champion.

\section{Corpus}

Cyrano [de] Bergerac, Savinien de, [1662] 2004, Les États et Empires de la Lune et du Soleil, édition critique de Madeleine Alcover, Paris, Champion Classiques.

Descartes, René, [1637] 1996, Discours de la méthode, EEuvres de Descartes, publiées par C. Adam et P. Tannery, nouvelle présentation par P. Costabel et B Rochot, 1964-1974, Vrin-CNRS.

Montaigne, Michel de, [1580] 2009, Essais, édition d’E. Naya, D. Reguig et A. Tarrête, Paris, Gallimard.

Vercors, 1952, Les Animaux dénaturés, Paris, Albin Michel.

\section{NOTES}

1. À l'époque de Cyrano on discute de la nature des anges et des êtres intermédiaires, qui sont loin, dans ce contexte religieux et culturel, d'être considérés comme des 
" êtres de fantaisie »; à preuve le succès du traité d'Apulée De deo Socratis, dont des traductions sont disponibles dès le début du siècle.

2. Comme le rossignol est un symbole du poète lyrique, la pie et le perroquet sont convoqués depuis l'Antiquité pour distinguer l'imitation d'un véritable langage.

3. Il s'agit ici d'une tradition ancienne que l'on trouve dès l'Antiquité et qui a été synthétisée par Porphyre dans le livre III du traité De l'abstinence.

4. «Et ce petit inconsolable me répondait dans les siens [airs de chanson], qu'il accepterait avec joie toute l'estime que je faisais de lui, s'il savait qu'elle lui pût faire mériter l'honneur de mourir à ma place ; mais que la Fortune n'ayant pas réservé tant de gloire à un malheureux comme lui, il acceptait de cette estime seulement ce qu'il en fallait pour m'empêcher de rougir de mon amitié » (Cyrano : 242).

5. Pour une approche globale de la question, voir Torero-Ibad (2009) et Gengoux (2015).

6. Montaigne : 195.

7. Je suis ici entièrement redevable au travail de Thierry Gontier.

8. «Les merles, les corbeaux, les pies, les perroquets, nous leur apprenons à parler : et cette facilité, que nous reconnaissons à nous fournir leur voix et haleine si souple et si maniable, pour la former et l'étreindre à certain nombre de lettres et de syllabes, témoigne qu'ils ont un discours au dedans. »

9. Sur la question des bruits, voir Laugaa (1973).

10. Cet aspect se retrouve aussi dans Les Oiseaux d'Aristophane, lorsque le chœur des Oiseaux célèbre leur propre gloire (v.676 et suiv.) et se moque de la condition misérable des «sans-ailes » [aptèn]. Ce passage n'est pas sans rappeler la prosopopée de l'oison formulée par Montaigne et dont la perspective relativiste cherchait à ébranler l'anthropocentrisme: "Car pourquoy ne dira un oison ainsi: Toutes les pieces de l'univers me regardent ; la terre me sert à marcher, le Soleil à m'esclairer, les estoilles à m'inspirer leurs influances [...] » (Essais, II, 12).

11. Les philosophes du Soleil, d'ailleurs, «ne parlent pas avec la langue » (Cyrano : 330), sans l'organe mais directement avec le cerveau, le foie et le cœur. Comme le montre Bérengère Parmentier, ce "langage sans médiation est un langage du corps dont l'explication est résolument matérialiste : les "idées" et "conceptions" logées dans les parties du corps sont si parfaitement matérielles qu'elles peuvent devenir lisibles " (Parmentier $2004: 219$ ).

\section{RÉSUMÉS}

Si l'antispécisme est un concept forgé au $\mathrm{Xx}^{\mathrm{e}}$ siècle, sa portée polémique et paradoxale émerge déjà $\mathrm{au} \mathrm{XVI} \mathrm{I}^{\mathrm{e}}$ et plus encore au XVII ${ }^{\mathrm{e}}$ siècle, sous la plume notamment de Cyrano de Bergerac dans Les États et Empires de la Lune et du Soleil. La fiction qui est au principe du voyage imaginaire, soutenue par une imagination subversive et affranchie de toutes contraintes, ouvre ainsi le champ des possibles en nous donnant à rencontrer des oiseaux qui parlent. Mais nous sommes loin ici du cadre apparemment convenu de l'apologue, et faire des animaux de véritables 
personnages littéraires, à l'instar des hommes, est porteur de sens. En effet, conférer un logos à l'animal est un acte paradoxal et subversif au XVII siècle, car il remet en cause les opinions communément admises - héritées de l'aristotélisme et du christianisme - sur la hiérarchie des êtres et sur la suprématie de l'homme au sein du vivant. Faire parler l'animal, c'est donc d'abord forcer le lecteur à décentrer son regard. La fiction nous donne à voir ce que la réalité nous dérobe, l'intériorité animale : non seulement les animaux sont doués de parole, et donc de raison, mais ils possèdent aussi une âme, deux attributs dont les hommes, depuis l'Antiquité, s'étaient attribué l'exclusivité. Faire parler l'animal comme l'homme, c'est donc d'abord pousser celui-ci à reconnaitre que l'animal est un homme comme les autres. À cet égard, le discours animal chez Cyrano imbrique des enjeux d'une part narratifs, stylistiques et énonciatifs, et, de l'autre, polémiques, philosophiques et éthiques.

"Antispeciesism" is a concept forged in the twentieth century, but its polemical and paradoxical scope already emerged in the sixteenth and even more so in the seventeenth century, notably under the pen of Cyrano de Bergerac in Les États et Empires de la Lune et du Soleil. Fiction, which is at the core of his imaginary journey, supported by a subversive imagination freed from any constraint, opens up the field of possibilities allowing us to meet talking birds. But here we are far from the apparently agreed framework of the apologue, for making animals true literary characters, like men, is meaningful. Indeed, conferring logos on an animal is a paradoxical and subversive act in the seventeenth century, as it calls into question commonly accepted opinionsinherited from Aristotelianism and Christianity-on the hierarchy of beings and the supremacy of man within the living world. Making the animal speak first leads the reader to shift his gaze. Fiction shows us what reality robs us of, that is, animal interiority: not only are animals gifted with speech, and therefore with reason, but they do also possess a soul, two attributes that men, since antiquity, have attributed to themselves exclusively. To have the animal speak like a man is first of all to push man to recognise that the animal is a man like any other. In this respect, Cyrano's animal discourse interweaves narrative, stylistic and enunciative issues on the one hand, with polemical, philosophical and ethical ones on the other.

\section{INDEX}

Keywords : Cyrano de Bergerac (Savinien de), animal, fiction, libertinism, fight against speciesism

Mots-clés : Cyrano de Bergerac (Savinien de), animal, fiction, libertinage, anti-spécisme

\section{AUTEUR}

\section{AUDE VOLPILHAC}

Université catholique de Lyon, France, UR « Confluence, Sciences et Humanités » 